\title{
ON TRIGONOMETRIC SERIES WITH GAPS
}

\author{
SHIGERU TAKAHASHI
}

(Recieved April 26, 1965)

1. Introduction. In [2] R. Salem and A. Zygmund have proved the central limit theorem of the theory of probability for lacunary trigonometric series. Recently, P. Erdös attempted to weaken the lacunarity assumption in their result. In fact he proved the

THEOREM [1]. Let $S_{N}(t)=\sum_{k=1}^{N} \cos 2 \pi n_{k}\left(t+\phi_{k}\right)$, where $\left\{\phi_{k}\right\}$ be an arbitrary sequence of real numbers and $\left\{n_{k}\right\}$ a sequence of positive integers satisfying the following gap condition;

$$
n_{k+1}>n_{k}\left(1+c_{k} / \sqrt{k}\right), \text { for } k \geqq 1 \text {, where } \lim _{k \rightarrow \infty} c_{k}=+\infty \text {. }
$$

Then we have, for any real number $x$,

$$
\left.\lim _{N \rightarrow \infty}\left|\left\{t ; 0 \leqq t \leqq 1, S_{N}(t) \leqq x \sqrt{N / 2}\right\}\right|=(2 \pi)^{-1 / 2} \int_{-\infty}^{x} e^{-u^{2} /} d u *\right)
$$

In that paper he pointed out that his theorem is also best possible in the following sense: To every positive constant $c$ there exists a sequence of integers $\left\{n_{k}\right\}$ for which $n_{k+1}>n_{k}(1+c / \sqrt{k})$ but (1.2) is not true.

The purpose of the present note is to prove the

THEOREM. Let $S_{N}(t)=\sum_{k=1}^{N} a_{k} \cos 2 \pi n_{k}\left(t+\phi_{k}\right)$, where $\left\{n_{k}\right\}$ be a sequence of positive integers satisfying the gap condition (1.1) and $\left\{a_{k}\right\},\left\{\phi_{k}\right\}$ arbitrary sequences of real numbers for which

$$
\left.A_{N}=\left(2^{-1} \sum_{k=1}^{N} a_{k}^{2}\right)^{1 / 2} \rightarrow+\infty \text {, and }\left|a_{N}\right|=O\left(A_{N} / \sqrt{N}\right) \text {, as } N \rightarrow+\infty * *\right) \text {. }
$$

*) For any measurable set $E,|E|$ denotes its Lebesgue measure.

**) For example the conditions are satisfied when $\left\{a_{k}\right\}$ is non-increasing and $\sum_{k=1}^{\infty} a_{k}^{z}=+\infty$. 
Then we have, for any set $E \subset[0,1]$ of positive measure and any real $x$,

$$
\lim _{N \rightarrow \infty}\left|\left\{t ; t \in E, S_{N}(t) \leqq x A_{N}\right\}\right| /|E|=(2 \pi)^{-1 / 2} \int_{-\infty}^{x} e^{-u^{2} / 2} d u
$$

From the above theorem we can easily obtain the

COROLLARY. Under the hypotheses of the preceding theorem it is seen that $\varlimsup_{N \rightarrow \infty}\left|\sum_{k=1}^{N} a_{k} \cos 2 \pi n_{k}\left(t+\phi_{k}\right)\right|=+\infty$, almost everywhere in $t$.

Our proof of the theorem is due to the following version of the central limit theorem for trigonometric series not necessarily lacunary.

THEOREM [3]. Let $S_{N}(t)$ be the $N$-th partial sum of a trigonometric series $\sum_{k=1}^{\infty} a_{k} \cos 2 \pi k\left(t+\phi_{k}\right)$ and $A_{N}=\left(2^{-1} \sum_{k=1}^{N} a_{k}^{2}\right)^{1 / 2}$, then we put

$$
\triangle_{k}(t)=S_{2^{k+1}}(t)-S_{2^{k}}(t), \text { and } B_{N}=A_{2^{N+1}} .
$$

Suppose if

$$
A_{N} \rightarrow+\infty, \quad \max _{t}\left|\triangle_{N}(t)\right|=o\left(B_{N}\right), \quad \text { as } \quad N \rightarrow \infty,
$$

and, for some function $g(t)$,

$$
\lim _{N \rightarrow \infty} \int_{0}^{1}\left|B_{N}^{-2} \sum_{k=1}^{N}\left\{\triangle_{k}^{2}(t)+2 \triangle_{k}(t) \triangle_{k+1}(t)\right\}-g(t)\right| d t=0,
$$

then the function $g(t)$ is non-negative and we have, for any set $E \subset[0,1]$ of positive measure and any real number $x \neq 0$,

$$
\lim _{N \rightarrow \infty}\left|\left\{t ; t \in E, S_{N}(t) / A_{N} \leqq x\right\}\right| /|E|=(2 \pi)^{-1 / 2}|E|^{-1} \int_{E} d t \int_{-\infty}^{\lambda(x, t)} e^{-u^{2,2}} d u
$$

where $\lambda(x, t)=x / \sqrt{g(t)}$ and $x / 0$ denotes $+\infty($ or $-\infty)$ if $x>0($ or $x<0)$.

In this theorem if $g(t)=1$ on some set of positive measure, then the values of $\left\{S_{N}(t) / A_{N}\right\}$ are distributed asymptotically normally on this set. 
2. Proof of the Theorem. To simplify the computations we will work out the proof of the theorem only for cosine series, the proof of the general case follows the same lines. Further without loss of generality, we may assume that the sequence of positive numbers $\left\{c_{k}\right\}$ in (1.1) is non-decreasing*). That is, we prove the theorem for trigonometric series $S_{N}(t)=\sum_{k=1}^{N} a_{k} \cos 2 \pi n_{k} t$ under the conditions :

(2. 1) $A_{N}=\left(2^{-1} \sum_{k=1}^{N^{2}} a_{k}^{2}\right)^{1 / 2} \rightarrow+\infty$, and $\left|a_{N}\right|=O\left(A_{N} / \sqrt{N}\right)$, as $N \rightarrow+\infty$, and

$\left(2.1^{\prime}\right) \quad n_{k+1}>n_{k}\left(1+c_{k} / \sqrt{k}\right)$, for $k \geqq 1$, and $c_{k} \uparrow+\infty$, as $k \rightarrow+\infty$.

I. First let us put, for $k=1,2, \cdots$,

$$
p(k)=\max \left\{m ; n_{m} \leqq 2^{k}\right\}
$$

and

$$
\left.\triangle_{k}(t)=\sum_{m=p(k)+1}^{p(k+1)} a_{m} \cos 2 \pi n_{m} t, \text { and } B_{k}=A_{p(k+1)} .^{* *}\right)
$$

If $p(k)+1<p(k+1)$, then $\left(2.1^{\prime}\right)$ implies that

$$
2>\prod_{m=p(k)+1}^{p(k+1)-1}\left(1+c_{m} / \sqrt{m}\right)>1+\{p(k+1)-p(k)-1\} c_{p(k)} / \sqrt{p(k+1)} .
$$

By $\left(2.1^{\prime}\right)$ and the above relation, we have always

(2. 3) $\quad p(k+1)-p(k)=o(\sqrt{p(k)})$ and $p(k+1) / p(k) \rightarrow 1$, as $k \rightarrow+\infty$.

Therefore, we obtain

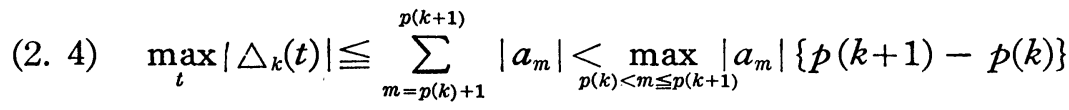

$$
=O\left(B_{k}\{p(k+1)-p(k)\}\{p(k)\}^{-1 / 2}\right)=o\left(B_{k}\right), \text { as } k \rightarrow+\infty \text {. }
$$

*) If $\left\{c_{k}\right\}$ is not non-decreasing, we use $\inf _{n \geqq k} c_{n}$ instead of $c_{k}$.

**) If $p(k)=p(k+1)$, then we put $\triangle_{k}(t) \stackrel{n \geqq k}{=0}$. 
II. If we put

$$
\left\{\begin{array}{l}
U_{k}(t)=\sum_{m=p(k)+2}^{p(k+1)} a_{m} \sum_{j=p(k)+1}^{m-1} a_{j} \cos 2 \pi\left(n_{m}-n_{j}\right) t, \\
V_{k}(t)=2^{-1} \sum_{m=p(k)+1}^{p(k+1)} a_{m} \sum_{j=p(k)+1}^{p(k+1)} a_{j} \cos 2 \pi\left(n_{m}+n_{j}\right) t,
\end{array}\right.
$$

then we have

$$
\triangle_{k}^{2}(t)-\left\|\triangle_{k}\right\|^{2}=U_{k}(t)+V_{k}(t) *{ }^{*}
$$

Then (2.5) and (2.4) imply that

$$
\left\|V_{k}\right\| \leqq\left\|\triangle_{k}\right\| \sum_{m=p(k)+1}^{p(k+1)}\left|a_{m}\right|=o\left(B_{k}\left\|\triangle_{k}\right\|\right), \quad \text { as } \quad k \rightarrow+\infty .
$$

Since $\left\{V_{k}(t)\right\}$ is orthogonal, we have

$$
\left\|\sum_{k=1}^{N} V_{k}\right\|^{2}=\sum_{k=1}^{N}\left\|V_{k}\right\|^{2}=o\left(\sum_{k=1}^{N} B_{k}^{2}\left\|\triangle_{k}\right\|^{2}\right)=o\left(B_{N}^{4}\right), \quad \text { as } \quad N \rightarrow+\infty .
$$

Further we have, by (2.4) and the above relations,

$$
\left\|U_{k}\right\| \leqq\left\|\triangle_{k}^{2}\right\|+\left\|V_{k}\right\| \leqq \max _{t}\left|\triangle_{k}(t)\right|\left\|\triangle_{k}\right\|+\left\|V_{k}\right\|=o\left(B_{k}\left\|\triangle_{k}\right\|\right) \text {, as } k \rightarrow+\infty .
$$

Hence we have, by $\left(2.5^{\prime}\right)$ and the above inequalities,

$$
\begin{aligned}
& \left\|\sum_{k=1}^{N}\left\{\triangle_{k}^{2}(t)-\left\|\triangle_{k}\right\|^{2}\right\}\right\|^{2} \leqq 2\left\|\sum_{k=1}^{N} U_{k}\right\|^{2}+2\left\|\sum_{k=1}^{N} V_{k}\right\|^{2} \\
& =4 \sum_{k=2}^{N} \sum_{j=1}^{k-1} \int_{0}^{1} U_{k}(t) U_{j}(t) d t+o\left(B_{N}^{4}\right), \text { as } N \rightarrow+\infty .
\end{aligned}
$$

III. In this section let us assume that for fixed $k>j$,

$$
p(j)+1<p(j+1) \text { and } p(k)+1<p(k+1)
$$

which means that both of $U_{j}(t)$ and $U_{k}(t)$ are not empty-sums. Then for any

*) For any $f(t) \in L^{2}(0,1),\|f\| \operatorname{denotes}\left\{\int_{0}^{1} f^{2}(t) d t\right\}^{1 / 2}$. 
fixed indexes $q$ and $h$ such that

$$
p(j)<h \leqq p(j+1) \text { and } p(k)<q \leqq p(k+1)
$$

we consider the number of solutions $\left(n_{r}, n_{i}\right)$ of the equation

$$
n_{q}-n_{r}=n_{l}-n_{i},
$$

where $p(k)<r<q$ and $p(j)<i<h$. From (2.8') and (2.8) it is seen that

$$
n_{r}=n_{q}-\left(n_{h}-n_{i}\right)>n_{q}-2^{j}>n_{q}\left(1-2^{j-k}\right) \geqq n_{q}\left(1+2^{j-k+1}\right)^{-1} .
$$

Therefore, the $n_{r}$ 's in $\left(2.8^{\prime}\right)$ must satisfy the following inequalities

$$
n_{q}\left(1+2^{j-k+1}\right)^{-1}<n_{r}<n_{q} \text { and } p(k)<r<p(k+1) .
$$

If $m_{1}$ (or $m_{2}$ ) denotes the minimum (or maximum) index of $n_{r}$ 's satisfying the above inequalities, then (2.1') implies that

$$
\begin{aligned}
\left(1+2^{j-k+1}\right) & >n_{m_{2}+1} / n_{m_{1}}>\prod_{m=m_{1}}^{m_{2}}\left(1+c_{m} / \sqrt{m}\right) \\
& >1+\left(m_{2}-m_{1}+1\right) c_{p(k)} / \sqrt{p^{(k+1)}} .
\end{aligned}
$$

By the above relation, we have $\left(m_{2}-m_{1}+1\right) \leqq 2^{j-k+1} c_{p(k)}^{-1} \sqrt{p(k+1)}$. Therefore, by (2.3) the number of $n_{r}$ 's in $\left(2.8^{\prime}\right)$ is at most $2^{j-k} C \sqrt{p(k)}$, where $C$ is a positive constant. Further for any given $q, r$ and $h$ there exists at most one $n_{i}$ satisfying $\left(2.8^{\prime}\right)$. Hence we can conclude that

$$
\left\{\begin{array}{l}
\text { for any given } q \text { and } h, \text { the number of solutions } \\
\left(n_{r}, n_{i}\right) \text { of }\left(2.8^{\prime}\right) \text { is at most } 2^{j-k} C \sqrt{p(k)}
\end{array}\right.
$$

IV. By (2.9) we have, for any given $q$ and $h$ satisfying (2.8),

$$
\begin{gathered}
\left|\int_{0}^{1} \sum_{r=p(k)+1}^{q-1} a_{r} \cos 2 \pi\left(n_{q}-n_{r}\right) t \sum_{i=p(j)+1}^{h-1} a_{i} \cos 2 \pi\left(n_{h}-n_{i}\right) t d t\right| \\
\left.\leqq C\left(\max _{p(k)<r<p(k+1)}\left|a_{r}\right|\right) \underset{p(j)<i<p(j+1)}{\max }\left|a_{i}\right|\right) 2^{j-k} \sqrt{p(k)},
\end{gathered}
$$

and by (2.1) and (2.3), the last term is not greater than $2^{j-k} C^{\prime} B_{k} B_{j} / \sqrt{p(j)+1}$ 
for some positive constant $C^{\prime}$. Therefore by (2.5) we have, for $k>j$,

$$
\int_{0}^{1} U_{k}(t) U_{j}(t) d t \leqq 2^{j-k} C^{\prime} B_{k}^{\prime \prime}\{p(j)+1\}^{-1 / 2}\left(\sum_{q=p(k)+2}^{p(k+1)}\left|a_{q}\right|\right)\left(\sum_{h=p(j)+2}^{p(j+1)}\left|a_{h}\right|\right) .
$$

Since we have

$\sum_{l=p(m)+2}^{p(m+1)}\left|a_{l}\right| \leqq \sqrt{2}\left\|\triangle_{m}\right\|\{p(m+1)-p(m)\}^{1 / 2}=o\left(\left\|\triangle_{m}\right\|\{p(m)\}^{1 / 4}\right)$, as $m \rightarrow+\infty$,

it is seen that

$$
\begin{aligned}
& \sum_{k=2}^{N} \sum_{j=1}^{k-1}\left|\int_{0}^{1} U_{k}(t) U_{j}(t) d t\right| \\
& \quad=o\left(B_{N}^{2}\right) \sum_{k=2}^{N}\left\|\triangle_{k}\right\|\{p(k)\}^{1 / 4} \sum_{j=1}^{k-1} 2^{j-k}\left\|\triangle_{j}\right\|\{p(j)+1\}^{-1 / 4} \text {, as } N \rightarrow+\infty .
\end{aligned}
$$

On the other hand by (2.3), we can find a constant $k_{0}$ such that

$$
2^{j-1-k}\{p(j-1)+1\}^{-1 / 2}<(2 / 3) 2^{j-k}\{p(j)+1\}^{-1 / 2}, \text { for } j>k_{0} \text {. }
$$

Hence we have

$$
\begin{aligned}
\sum_{j=1}^{k-1} 2^{j-k}\{p(j)+1\}^{-1 / 2} & <\sum_{j=1}^{k_{0}-1} 2^{j-k}+\sum_{j=k_{0}}^{k} 2^{j-k}\{p(j)+1\}^{-1 / 2} \\
& <2^{k_{0}-k}+\{p(k)+1\}^{-1 / 2} \sum_{j=k_{0}}^{k}(2 / 3)^{k-j}
\end{aligned}
$$

Further from (2.1') and (2.2) it is easily seen that $p(k)=O\left(k^{2}\right)$, as $k \rightarrow+\infty$. Therefore, we can find a constant $K$ such that

$$
\sum_{j=1}^{k-1} 2^{j-k}\{p(j)+1\}^{-1 / 2}<K\{p(k)+1\}^{-1 / 2}, \text { for all } k .
$$

Hence by the Schwarz inequality and the above relation, we have

$$
\sum_{j=1}^{k-1} 2^{j-k}\left\|\triangle_{j}\right\|\{p(j)+1\}^{-1 / 4} \leqq K\left(\sum_{j=1}^{k-1} 2^{j-k}\left\|\triangle_{j}\right\|^{2}\right)^{1 / 2}\{p(k)+1\}^{-1 / 4},
$$

and this implies that 
(2. 10)

$$
\begin{aligned}
\sum_{k=2}^{N} \sum_{j=1}^{k-1}\left|\int_{0}^{1} U_{k}(t) U_{j}(t) d t\right|=o\left(B_{N}^{2}\right) \sum_{k=2}^{N}\left\|\triangle_{k}\right\|\left(\sum_{j=1}^{k-1} 2^{j-k}\left\|\triangle_{j}\right\|^{2}\right)^{1 / 2} \\
=o\left(B_{N}^{3}\right)\left(\sum_{k=2}^{N} \sum_{j=1}^{k-1} 2^{j-k_{\|}}\left\|\triangle_{j}\right\|^{2}\right)^{1 / 2}=o\left(B_{v}^{4}\right), \text { as } N \rightarrow+\infty .
\end{aligned}
$$

V. If we put

$$
\left\{\begin{array}{l}
G_{k}(t)=2^{-1} \sum_{q=p(k+1)+1}^{p(k+2)} a_{q} \sum_{r=p(k)+1}^{p(k+1)} a_{r} \cos 2 \pi\left(n_{q}-n_{r}\right) t, \\
H_{k}(t)=2^{-1} \sum_{q=p(k+1)+1}^{p(k+2)} a_{q} \sum_{r=p(k)+1}^{p(k+1)} a_{r} \cos 2 \pi\left(n_{q}+n_{r}\right) t,
\end{array}\right.
$$

then we have, by (2.4),

$$
\left.\left\|G_{k}\right\|=o\left(B_{k}\left\|\triangle_{k}\right\|\right) \text { and }\left\|H_{k}\right\|=o\left(B_{k}\left\|\triangle_{k}\right\|\right), \text { as } k \rightarrow+\infty *\right) .
$$

Since $G_{k}(t)+H_{k}(t)=\triangle_{k}(t) \triangle_{k+1}(t)$ and $\left\{H_{k}(t)\right\}$ is orthogonal, we have in the same way as (2.6),

$$
\left\|\sum_{k=1}^{N} \triangle_{k}(t) \triangle_{k+1}(t)\right\|^{2}=4 \sum_{k=3}^{N} \sum_{j=1}^{k-2} \int_{0}^{1} G_{k}(t) G_{j}(t) d t+o\left(B_{N}^{4}\right), \text { as } N \rightarrow+\infty .
$$

Further by the same arguments as we used to obtain (2.10), it is seen that

$$
\sum_{k=3}^{N} \sum_{j=1}^{k-2}\left|\int_{0}^{1} G_{k}(t) G_{j}(t) d t\right|=o\left(B_{N}^{4}\right), \quad \text { as } \quad N \rightarrow+\infty .
$$

Therefore, we have

$$
\left\|B_{N}^{-2} \sum_{k=1}^{N^{*}} \triangle_{k}(t) \triangle_{k+1}(t)\right\|=o(1), \text { as } \quad N \rightarrow+\infty .
$$

VI. Combining (2.6), (2.10) and (2.11) we have

$$
\left\|B_{N}^{-2} \sum_{k=1}^{N}\left\{\triangle_{k}^{\mathscr{k}}(t)+2 \triangle_{k}(t) \triangle_{k+1}(t)\right\}-1\right\|=o(1), \text { as } N \rightarrow+\infty .
$$

*) It is easily seen that $B_{N+1} / B_{N} \rightarrow 1$, as $N \rightarrow+\infty$. 
By (2.4) and (2.12) we can apply the last theorem in $\$ 1$ to this trigonometric series for which we have obtained $g(t)=1$. This completes the proof.

3. Proof of the Corollary. Let us put $S_{N}(t)=\sum_{k=1}^{N} a_{k} \cos 2 \pi n_{k}\left(t+\phi_{k}\right)$ and assume that $\varlimsup_{N \rightarrow \infty}\left|S_{N}(t)\right|<+\infty$ on some set of positive measure. Then there exists a positive number $M$ such that $\left|\left\{t ; 0 \leqq t \leqq 1, \varlimsup_{N \rightarrow \infty}\left|S_{N}(t)\right|<M\right\}\right|>0$. Since $\left\{t ; 0 \leqq t \leqq 1, \varlimsup_{N \rightarrow \infty}\left|S_{N}(t)\right|<M\right\} \subset \bigcup_{n=1}^{\infty} \bigcap_{N=n}^{\infty}\left\{t ; 0 \leqq t \leqq 1,\left|S_{N}(t)\right|<M\right\}$, there exist a positive integer $N_{0}$ and a set $E \subset[0,1]$ such that

$$
|E|>0 \text { and }\left|S_{N}(t)\right|<M \text {, if } t \in E \text { and } N>N_{0} .
$$

On the other hand from our theorem it is seen that

$$
\lim _{N \rightarrow \infty}\left|\left\{t ; t \in E,\left|S_{N}(t)\right|<M\right\}\right|=0 .
$$

This contradiction completes the proof.

Finally, the author thanks Professor P. Erdös for his kindness.

\section{REFERENCES}

[1] P. ERDös, On trigonometric sums with gaps, Magyar Tud. Akad. Kutatós. Int. Közl., 7(1962), 37-42.

[2] R. SAlem AND A. ZYGmund, On lacunary trigonometric series, I, II, Proc. Nat. Acad. Sci. U.S. A., 33(1947), 333-338; ibid. 34(1948), 54-62.

[3] S. TAKAHASHI, A version of the central limit theorem for trigonometric series, Tohoku Math. Journ., 16(1964), 384-398.

\section{DEPARTMENT OF MATHEMATiCs} KANAZAWA UNIVERSITY. 